\title{
Blow-up for wave equation with the scale-invariant damping and combined nonlinearities
}

\author{
Makram Hamouda ${ }^{1}$ and M.Ali Hamza ${ }^{1}$ \\ ${ }^{1}$ Imam Abdulrahman Bin Faisal University
}

June 27, 2020

\begin{abstract}
In this article, we study the blow-up of the damped wave equation in the $\backslash$ textit $\{$ scale-invariant case $\}$ and in the presence of two nonlinearities. More precisely, we consider the following equation: \begin } \{ \text { displaymath } \} \backslash \mathrm { d } _ { \mathrm { u } _ { - } } \{ \mathrm { tt } \} - \backslash \operatorname { D e l t a } \mathrm { u } + \backslash \mathrm { frac } \{ \backslash \mathrm { mu } \} \{ 1 + \mathrm { t } \} \mathrm { u } _ { - } $\mathrm{t}=\left|\mathrm{u}_{-} \mathrm{t}\right|^{\wedge} \mathrm{p}+|\mathrm{u}|^{\wedge} \mathrm{q}, \backslash$ quad $\backslash \operatorname{mbox}\{\operatorname{in}\} \backslash \backslash \mathrm{R}^{\wedge} \mathrm{N} \backslash$ times[0, infty), \end\{displaymath } \} \text { with small initial data. } \backslash \backslash \text { For } \$ \backslash \mathrm { mu } < \backslash \text { frac } \{ \mathrm { N } ( \mathrm { q } 1) $\}\{2\} \$$ and $\$ \backslash \mathrm{mu} \backslash$ in $\left(0, \backslash m u_{-}^{*}\right) \$$, where $\$ \backslash m u_{-}^{*}>0 \$$ is depending on the nonlinearties' powers and the space dimension $\left(\$ \backslash \mathrm{mu}_{-} * \$\right.$ satisfies $\$(\mathrm{q}-1) \backslash \operatorname{left}\left(\left(\mathrm{N}+2 \backslash \mathrm{mu}_{-}{ }^{*}-1\right) \mathrm{p}-2 \backslash\right.$ right $\left.)=4 \$\right)$, we prove that the wave equation, in this case, behaves like the one without dissipation $(\$ \backslash \mathrm{mu}=0 \$)$. Our result completes the previous studies in the case where the dissipation is given by $\$ \backslash \operatorname{frac}\{\backslash \mathrm{mu}\}\left\{(1+\mathrm{t})^{\wedge} \backslash\right.$ beta\} $\}$ _t; $\backslash \backslash$ beta $>1 \$$ ((missing citation)), where, contrary to what we obtain in the present work, the effect of the damping is not significant in the dynamics. Interestingly, in our case, the influence of the damping term $\$ \backslash$ frac $\{\backslash \mathrm{mu}\}\{1+\mathrm{t}\} \mathrm{u}_{\mathrm{t}} \mathrm{t} \$$ is important.
\end{abstract}

\section{Hosted file}

Blow-up-Damped wave.pdf available at https://authorea.com/users/44799/articles/462953-blowup-for-wave-equation-with-the-scale-invariant-damping-and-combined-nonlinearities

\section{References}

